ARTICLE

\title{
Above-room-temperature ferroelectricity and antiferroelectricity in benzimidazoles
}

\author{
Sachio Horiuchi ${ }^{1,2}$, Fumitaka Kagawa ${ }^{2,3}$, Kensuke Hatahara ${ }^{3}$, Kensuke Kobayashi ${ }^{4}$, Reiji Kumai ${ }^{2,4}$, \\ Youichi Murakami ${ }^{4} \&$ Yoshinori Tokura 3,5
}

The imidazole unit is chemically stable and ubiquitous in biological systems; its proton donor and acceptor moieties easily bind molecules into a dipolar chain. Here we demonstrate that chains of these amphoteric molecules can often be bistable in electric polarity and electrically switchable, even in the crystalline state, through proton tautomerization. Polarization-electric field $(P-E)$ hysteresis experiments reveal a high electric polarization ranging from 5 to $10 \mu \mathrm{C} \mathrm{cm}^{-2}$ at room temperature. Of these molecules, 2-methylbenzimidazole allows ferroelectric switching in two dimensions due to its pseudo-tetragonal crystal symmetry. The ferroelectricity is also thermally robust up to $400 \mathrm{~K}$, as is that of 5,6-dichloro-2-methylbenzimidazole (up to $\sim 373 \mathrm{~K}$ ). In contrast, three other benzimidazoles exhibit double $P-E$ hysteresis curves characteristic of antiferroelectricity. The diversity of imidazole substituents is likely to stimulate a systematic exploration of various structure-property relationships and domain engineering in the quest for lead- and rare-metal-free ferroelectric devices.

\footnotetext{
${ }^{1}$ National Institute of Advanced Industrial Science and Technology (AIST), Tsukuba 305-8562, Japan. ${ }^{2}$ CREST, Japan Science and Technology Agency (JST), Tokyo 102-0076, Japan. ${ }^{3}$ Department of Applied Physics, University of Tokyo, Tokyo 113-8656, Japan. ${ }^{4}$ Condensed Matter Research Center (CMRC) and Photon Factory, Institute of Materials Structure Science, High Energy Accelerator Research Organization (KEK), Tsukuba 305-0801, Japan. ${ }^{5}$ Correlated Electron Research Group (CERG) and Cross-correlated Materials Research Group (CMRG), RIKEN Advanced Science Institute, Wako 351-0198, Japan. Correspondence and requests for materials should be addressed to S.H. (email: s-horiuchi@aist.go.jp).
} 
F erroelectricity, that is, electric polarity in solids or liquid crystals that is switchable with the electric field $E$, is in increasing demand as it provides diverse entries into electronic, electromechanical, optical and optoelectronic applications $^{1-3}$. In ferroelectrics, the spontaneous electric polarization $P$ at $E=0$ can be reversibly inverted by reversing the electric field, and the polarization-electric field $(P-E)$ curve then shows a hysteresis loop. The related antiferroelectricity phenomenon exhibits a double $P-E$ hysteresis curve when the dipolar arrangement is reversed stepwise through the antipolar arrangement at low electric field ${ }^{4}$. A very high polarization response, which is promising for capacitors, memories and piezoelectric applications, has so far been discovered mostly in inorganic oxides containing toxic lead or rare elements, such as bismuth, tantalum, niobium and so on. The high-performance piezoelectricity of lead-based ceramics is often related to competing ferroelectric and antiferroelectric orders ${ }^{3,5}$. To decrease our reliance on these materials and to work towards a green and sustainable society, purely organic ferroelectrics ${ }^{6,7}$, which are expected to be used as key materials in organic, printable and bendable electronic device applications, are being pursued as possible alternatives. Recent advances in piezoresponse force microscopy (PFM) have also revealed the nanoscale ferroelectricity of crystalline $\gamma$-form glycine and porcine aortic walls ${ }^{8,9}$. Here we demonstrate that imidazoles carrying large electric dipoles constitute a new family of singlecomponent ferroelectrics, as well as antiferroelectrics, in the bulk state with variable chemical substituents.

In an earlier supramolecular approach using acid and base molecules, proton donor and acceptor moieties were joined together to host the proton dynamics for switching the bistable polarities ${ }^{8}$. This principle was recently extended to singlecomponent compounds; the proton tautomerism on $\mathrm{O}-\mathrm{H} \cdots \mathrm{O}$ bonds achieves excellent room-temperature ferroelectricity in croconic acid ${ }^{7}, \beta$-diketone enols and carboxylic acids ${ }^{10}$. There are, however, some drawbacks specific to each compound for the fabrication of thin-film devices. For instance, croconic acid is corrosive and also requires a strong acid for crystallization. Some $\mathrm{O}-\mathrm{H} \cdots \mathrm{O}$-bonded organic ferroelectrics are unstable against air oxidation, whereas others are thermally fragile. Satisfying all the performance and process-compatibility demands together with sufficient electric, mechanical and environmental endurance is a challenging task.

The imidazole ring is an important building block in biological systems, as exemplified by histidine, histamine and vitamin $\mathrm{B}_{12}$ (refs 11-13). In addition, these compounds are now currently utilized as ingredients in various ionic liquids ${ }^{14}$, as well as a corrosion inhibitor for copper. Many derivatives are commercially available, stable in air and highly soluble in alcohols and other common organic solvents. The small imidazole molecule carries a large dipole moment $(3.61 \mathrm{D})$ directed nearly parallel to the $\mathrm{N}-\mathrm{H}$ bond. Imidazole can both donate and accept protons with modest acidity ${ }^{15}$, and this amphoteric nature has been exploited as a guest proton carrier in porous coordination polymers designed for anhydrous protonconductive materials working at high temperatures ${ }^{16}$. In the crystalline state, the chemical mechanism designated in Fig. 1a is anticipated for the polarization reversal. Unless the substituents allow for steric hindrance or distinct hydrogen bonding, imidazole moieties can spontaneously assemble into a linear chain structure because of the directional nature ${ }^{17}$ of the intermolecular $\mathrm{N}-\mathrm{H} \cdots \mathrm{N}$ bonds. The polarity of the chain can be inverted cooperatively by the concomitant $\pi$-bond switching and proton transfer that alters the $\mathrm{N}-\mathrm{H} \cdots \mathrm{N}$ bond to $\mathrm{N} \cdots \mathrm{H}-\mathrm{N}$ and vice versa. It should be noted that the scheme shown in Fig. 1a is generalized for various combinations of substituents $\mathrm{X}$,
$\mathrm{Y}_{1}$ and $\mathrm{Y}_{2}$. Therefore, the discovery of above-room-temperature ferroelectricity and antiferroelectricity that we report here will stimulate a systematic exploration of various structure-property relationships in the quest for lead- and rare-metal-free ferroelectric devices.

\section{Results}

Structural assessment. To scrutinize these chemical schemes, we focused first on the imidazoles of $Y_{1}=Y_{2}$ and their ring-fused analogues, benzimidazoles (Fig. 1b), because a number of related compounds have already been deposited in the Cambridge Structural Database. We searched the database for linear chain structures having some pseudo-symmetry to determine which candidate ferroelectrics have so far been overlooked, but we did not encounter this example in the literature. The 5,6-dichloro-2methylbenzimidazole (DC-MBI) crystal belongs to the orthorhombic system with the space group $P c a 2_{1}$ (no. 29). The polarity of all the hydrogen-bonded chains was found to align along the crystal $c$ direction (Fig. 1c). The global structure can be satisfactorily refined with slightly higher $R$ factors under the higher crystal symmetry of $\mathrm{Pbcm}$ (no. 57) by locating a mirror symmetry plane normal to the molecular plane. In this model, the protons inevitably occupy disordered or centred locations between two nitrogen atoms.

The alternative approach is to determine the disordered or centred location of the protons. If this crystal structure represents the paraelectric state, then the protons may be ordered as asymmetric $\mathrm{N}-\mathrm{H} \cdots \mathrm{N}$ bonds form at temperatures lower than the Curie point and host ferroelectricity or antiferroelectricity. The other possibility is the artefacts arising from a pseudo-symmetry element assumed in the analysis misled by the global structure of minute deviation from the higher symmetry. We found that this occurs in the 2-methylbenzimidazole (MBI) crystal. The reported MBI crystal structure ${ }^{18}$ belongs to the tetragonal system with the symmetric space group $P 4_{2} / n$ (no. 86). The protons are disordered over two sites, and their hydrogen bonds form straight bands running along either $[110]_{\text {tetra }}$ or the crystallographically equivalent $[1 \overline{1} 0]_{\text {tetra }}$ direction. In our analysis, the tetragonal lattice is barely lowered to the monoclinic system with the space group $P n$ (no. 7), which is one of the maximal subgroups of $P 4_{2} / n$ by interchanging the crystallographic $b$ and $c$ axes. Because the as-grown MBI crystals (Supplementary Fig. S1 and Supplementary Table S1) are not in a single-domain state but easily mix the tetragonal $a$ and $b$ axes, the axes and plane indices are presented here according to the tetragonal symmetry. The proton ordering is responsible for the polarity within the $(001)_{\text {tetra }}$ plane in the bulk (Fig. 1d).

Ferroelectric properties. We measured the $P-E$ hysteresis curves for both the MBI and DC-MBI crystals with the applied electric field parallel to the hydrogen-bonded molecular sequence direction: $E \|[110]_{\text {tetra }}$ for MBI and $E \|[001]$ for DC-MBI. At room temperature, the MBI crystal exhibited ferroelectricity with quasi-rectangular loops and a remanent polarization as large as $5.2 \mu \mathrm{Ccm}^{-2}$ (Fig. 2a). The coercive field determined from the $P=0$ intercept is $11 \mathrm{kV} \mathrm{cm}^{-1}$ at $0.2 \mathrm{~Hz}$ and increases with frequency. Symmetry breaking from the tetragonal lattice permits polarization switching with any electric field direction parallel to the $(001)_{\text {tetra }}$ plane. In fact, measurements with electric field configurations of $E \|[100]_{\text {tetra }}$ and orthogonal $E \|[010]_{\text {tetra }}$ applied separately to two pieces cut from the same single crystal confirmed the apparent isotropic value of the remanent polarization within the $(001)_{\text {tetra }}$ sheet $\left(3.9-4.0 \mu \mathrm{Ccm}^{-2}\right.$, see Supplementary Fig. S3a and Supplementary Discussion). The ferroelectricity was also confirmed at higher temperatures of up to $400 \mathrm{~K}$ from the $P-E$ hysteresis loops, which exhibited little change in the 
a

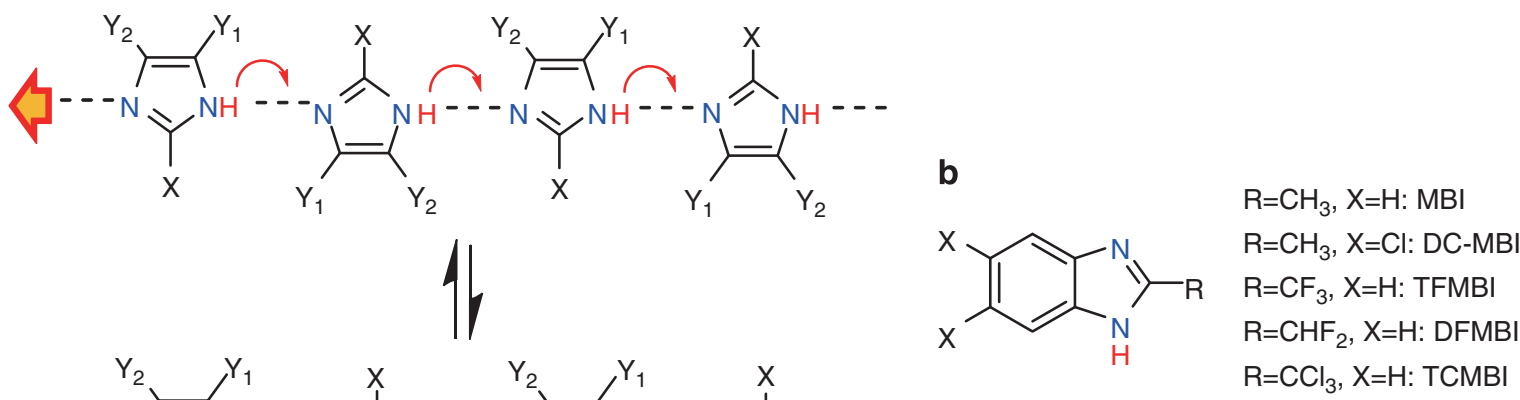

C

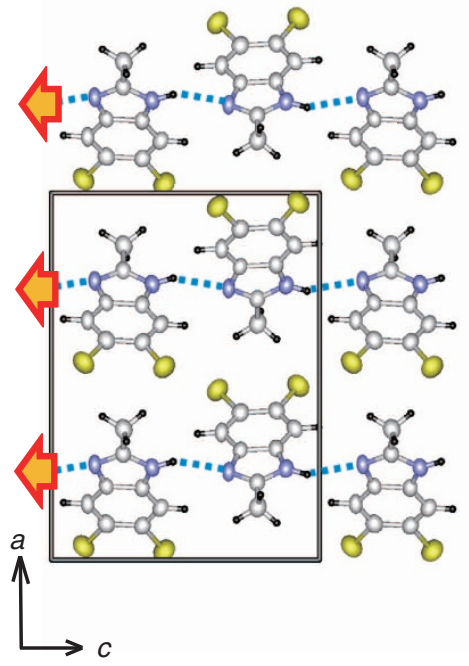

d

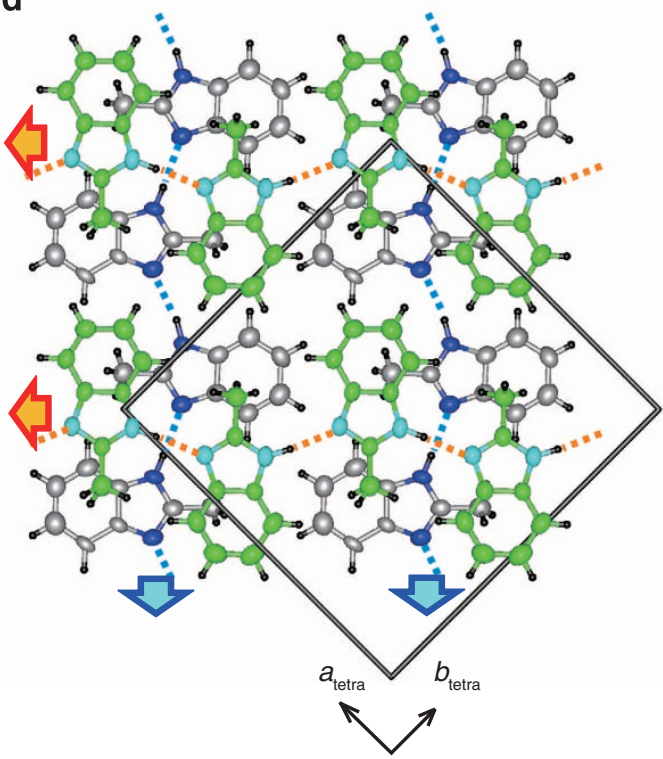

e

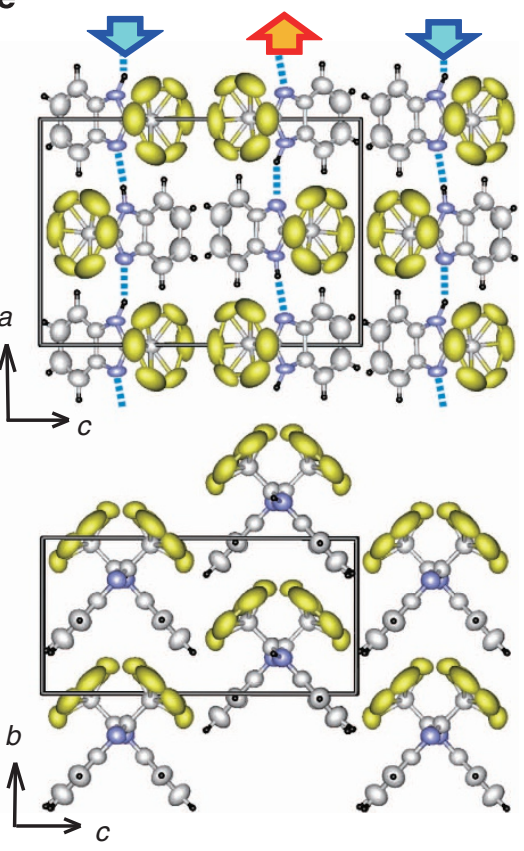

Figure 1 | Chemical and crystal structures of ferroelectric and antiferroelectric imidazoles. (a) Hydrogen bonding (broken lines) and polarization reversal mechanism through the proton tautomerism of the imidazole moiety. (b) Chemical structures of the ferroelectric and antiferroelectric benzimidazoles. Abbreviations are given for the imidazole derivatives. (c) Ferroelectric DC-MBI crystal viewed along the crystallographic $b$ direction. (d) Pseudo-tetragonal molecular arrangement and hydrogen-bonded sequences therein of the ferroelectric MBI. (e) Molecular arrangement of the antiferroelectric TCMBI crystal viewed along the crystallographic $b$ (top) and $a$ directions (bottom). The $\mathrm{CCl}_{3}$ group is in rotational disorder over two conformations.
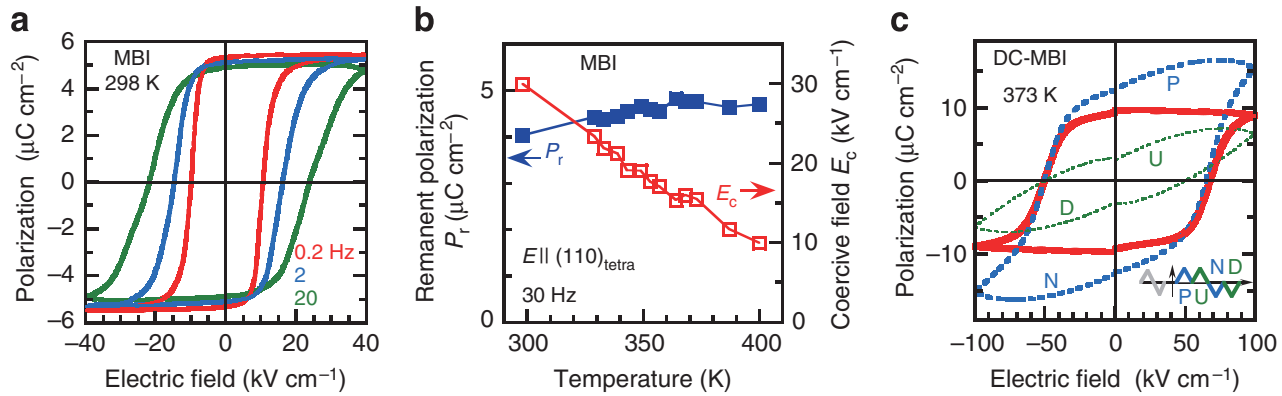

Figure 2 | Ferroelectric properties. (a) Electric polarization $(P)$ versus electric field $(E)$ hysteresis loops of $[110]_{\text {tetra }}$ polarization in $M B I$ measured at room temperature with a triangular ac electric field of various frequencies $(f=0.2-20 \mathrm{~Hz})$. (b) Temperature dependence of the remanent polarization and coercive field of $\mathrm{MBI}$ obtained from the $[110]_{\text {tetra }}$ hysteresis loops with $f=30 \mathrm{~Hz}$. (c) The dielectric hysteresis of the c-direction polarization in DC-MBI at $T=373 \mathrm{~K}$ measured using the double-wave method with a triangular ac electric field of $f=5 \mathrm{~Hz}$ (see inset for schematic of applied waveform). The pure hysteresis component (solid curve) was obtained by subtracting the 'non-hysteresis' polarization (up (U) and down (D) runs) from the total (positive (P) and negative $(\mathrm{N})$ runs). 
remanent polarization but did exhibit a decrease in the coercive field with temperature (Fig. 2b and Supplementary Fig. S3b).

The DC-MBI crystal exhibited a coercive field at room temperature that was too high to complete a satisfactory loop even with the maximum field of $100 \mathrm{kV} \mathrm{cm}^{-1}$. The hard switching likely reflects the elevated potential barrier for a proton to hop between the bistable potential minima because of the elongated $\mathrm{N} \cdots \mathrm{N}$ distance $(2.98 \AA)$ compared with that of MBI $(2.91 \AA$ on average). The spontaneous polarization was evaluated by reducing the coercive field at elevated temperatures. A hysteresis curve could be discerned at temperatures $>300 \mathrm{~K}$. Additional contributions from electrical conduction (leakage) through the sample were suggested by the longitudinally warped $P-E$ curves of the raw data, but this warping could be eliminated by the double-wave method ${ }^{19}$ by applying a double-triangular waveform voltage (see inset in Fig. $2 c$ for the applied waveform). The quasiparallelogram of the hysteresis contribution thus extracted from the data at $373 \mathrm{~K}$ gave a large remanent polarization of $10 \mu \mathrm{C} \mathrm{cm}^{-2}$ and coercive field of about $60 \mathrm{kV} \mathrm{cm}^{-1}$ (Fig. 2c).

Ferroelectric domain structure. The pseudo-tetragonal symmetry of MBI allows the relative orientation of the polarization in perpendicular $\left(90^{\circ}\right)$ configurations as well as conventional antiparallel $\left(180^{\circ}\right)$ configurations. To verify the multi-domain structure in the single crystal composed of both $90^{\circ}$ and $180^{\circ}$ domains, we employed PFM (Methods). The topography (Fig. 3a) of the cleaved $(001)_{\text {tetra }}$ surface shows a step-and-terrace profile with a step height of, predominantly, $c / 2$ or $c(\sim 7.2 \AA)$, reflecting the layered structure of MBI. The corresponding in-plane piezoresponse image is shown in Fig. $3 b$, where four kinds of ferroelectric domains are clearly discerned. A sequence of PFM measurements with a distinct cantilever geometry (see Supplementary Fig. S6) enabled us to identify the directions of the local polarizations $\boldsymbol{P}_{\text {local }}$, which were determined to be along any $[100],[-100],[010]$ or $[0-10]$ direction in the pseudotetragonal setting. This then demonstrates the existence of $90^{\circ}$ domain walls in terms of PFM, which is consistent with the apparent isotropic remanent polarization mentioned above. We note that the pattern of the observed domain configurations is richer than our original expectation; the prevailing $90^{\circ}$ domain walls often merge at a single point to form vortex and anti-vortex structures. Similar vortex structures have recently been reported in $\mathrm{BiFeO}_{3}$ thin films ${ }^{20}$, where the vortices are artificially created, or written, by scanning the cantilever with dc bias.

Antiferroelectric properties. The polarization was reversed at room temperature by applying an electric field parallel to the hydrogen-bonded chain for the other three imidazoles: 2-trifluoromethylbenzimidazole (TFMBI), 2-difluoromethylbenzimidazole (DFMBI) and 2-trichloromethylbenzimidazole (TCMBI) (see Fig. 1b), and their P-E curves, as shown in Fig. 4, are similar. At low electric fields, the polarization increases linearly with $E$, whereas quasi-step-like changes appear at higher fields with hysteresis. This double hysteresis loop is characteristic of antiferroelectricity, which appears when the free energy of an antipolar arrangement of dipolar chains is comparable to that of a polar arrangement. By subtracting the normal dielectric (linear $P-E)$ component, we obtained electrically induced polarizations of about 8,6 and $8 \mu \mathrm{Cm}^{-2}$ for TFMBI, DFMBI and TCMBI, respectively. Such large polarizations are comparable to the remanent polarization of DC-MBI, signalling the fully aligned polarity of the dipolar chains under an electric field beyond the critical field.

According to our crystal structural analysis, these antiferroelectrics have similar molecular and hydrogen-bonded chain structures (Supplementary Fig. S5). It should be noted that the large thermal ellipsoid over two sites for each halogen atom corresponds to the rotational disorder of the trihalomethyl groups
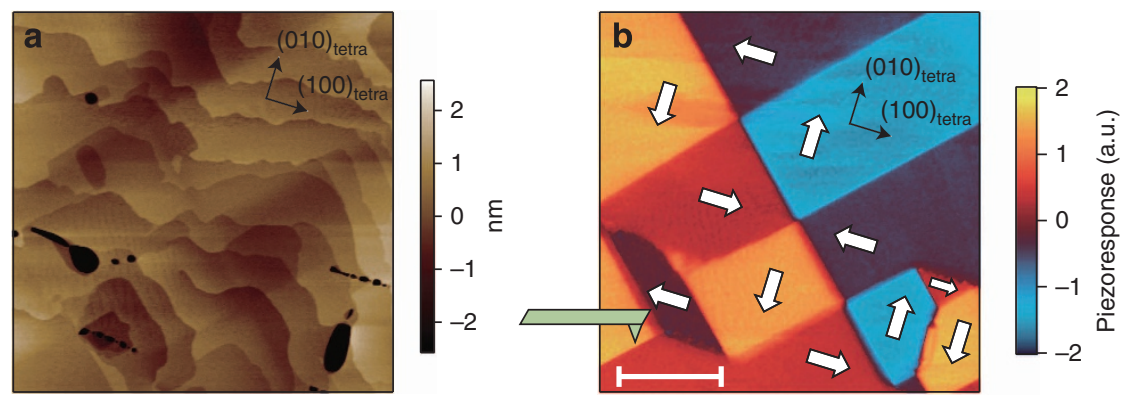

Figure 3 | PFM image of MBI single crystal. (a) Topography image of the cleaved [001] tetra surface. (b) In-plane piezoresponse image. The geometry of the cantilever is illustrated schematically, and the directions of the local polarizations are indicated by white arrows. The scale bar corresponds to $4 \mu \mathrm{m}$.
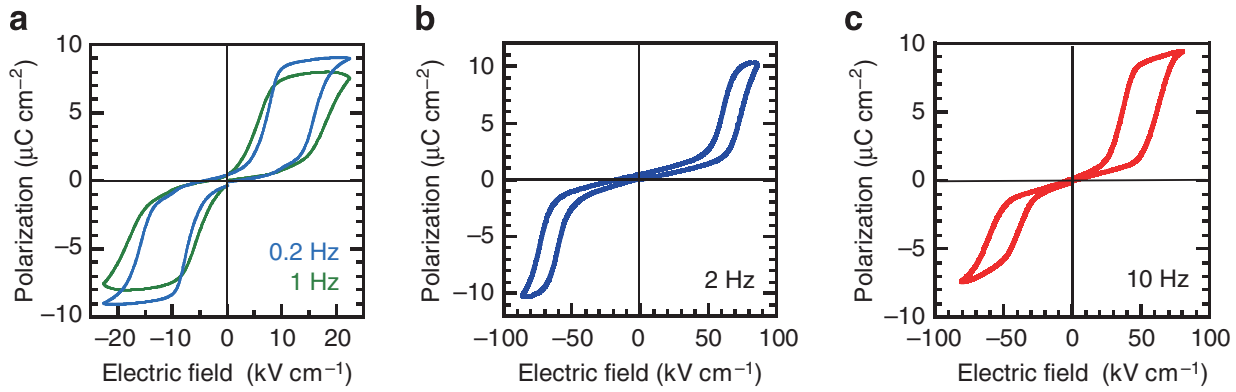

Figure 4 | Antiferroelectric properties at room temperature. The applied electric field was parallel to the direction of the hydrogen-bonded molecular sequence. (a) TFMBI, (b) DFMBI and (c) TCMBI crystals. Molecule abbreviations are given in Fig. $1 \mathrm{~b}$. 
in the TFMBI and TCMBI crystals. By ignoring the proton locations, these antiferroelectric molecules have pseudo-mirror symmetry normal to the molecular plane. In reality, both the molecular and crystal symmetries are lowered by the proton ordering. The three-dimensional molecular arrangements of these crystals are not isomorphous to one another. Although ferroelectricity requires a polar crystal structure, antiferroelectricity is usually related to the nonpolar structure at $E=0$, which is true for the TFMBI and DFMBI crystals, as they exhibit an antipolar arrangement of dipolar chains. Because of the proton ordering, the pseudo-orthorhombic lattice with the space group $\mathrm{Pbcm}$ (no. 57) of the TFMBI and DFMBI crystals is lowered to the monoclinic lattice with the symmetric space group $P 2_{1} / c$ (no. 14). In contrast, antiferroelectric TCMBI is a polar crystal (Fig. 1e); the pseudo-orthorhombic symmetry of the polar space group $P m c 2_{1}$ (no. 26) is lowered to that of the $P 2_{1}$ (no. 4) monoclinic lattice. The dipole moment component along the transverse molecular axes is arranged in an antiparallel manner, causing antiferroelectricity along the crystal $a$-axis, whereas that along the longitudinal axes cannot be cancelled out by the crystal symmetry, and permanent polarity is retained along the $b$ direction normal to the hydrogen-bonded chains.

Thermal properties. For all five compounds, the temperaturedependent permittivity measured along the dipolar chains simply increases with temperature at least up to $\sim 360-380 \mathrm{~K}$ (Supplementary Figs S2 and S4 and Supplementary Discussion) without any peak anomaly indicative of the Curie point. Thus, the ferroelectric or antiferroelectric phase persists from room temperature to far above room temperature. These compounds, except DC-MBI, did not display any phase transitions in differential scanning calorimetry (DSC) measurements until the samples gradually start to sublime at around $400-420 \mathrm{~K}$. For DC-MBI, we found a first-order phase transition with a large hysteresis at high temperature; exothermic and endothermic peaks appeared at 369 and $399 \mathrm{~K}$, respectively (Supplementary Fig. S4a).

\section{Discussion}

The discovery of ferroelectricity provides firm evidence for proton tautomerism, which comprises proton transfer and concomitant $\pi$-bond switching. This intriguing issue has long been studied in both the solution and solid states and also been addressed in relation to the large permittivity observed along the hydrogen-bonded chains of imidazole-related compounds ${ }^{21}$. The ac electric fields employed for the permittivity measurements are a few orders of magnitude smaller than that required for polarization reversal, and thus can probe only local fluctuations of the proton tautomerism and/or the bound motion of charged kink solitons on the hydrogen-bonded chains. As demonstrated for some benzimidazoles, this response may be tiny or even silent, regardless of the ferroelectricity or antiferroelectricity, especially when the Curie temperature is far above room temperature. In contrast, the reversal of a large polarization manifests collective switching that occurs in the whole crystal under a sufficiently large electric field. Incidentally, we note that in previous studies on the ferroelectricity of some imidazolium salts with inorganic anions ${ }^{22,23}$, it was reported that the ferroelectricity arises from the orientational order-disorder phenomena of both dipolar component ions, whereas completely protonated imidazolium cations cannot trigger collective proton dynamics.

In addition to the chemical and thermal stability, the imidazole moieties have some advantages in terms of their substituent diversity. We found that the linear hydrogen-bonded chains can be aligned in various three-dimensional architectures. This feature is quite promising for finding new paradigms with various structure-property relationships, for instance, such as crystal symmetry and dimensionality. Polarity switching over two dimensions as seen for MBI is significantly advantageous for improving the polarization performance of the randomly orientated polycrystalline state, such as the thin-film form, compared with the uniaxial polarity available in other organic ferroelectrics except TEMPO ${ }^{24}$ (tanane). An improved crystal symmetry also permits the formation of non- $180^{\circ}$ domains (that is, the $90^{\circ}$ domain for $\mathrm{MBI}$ ), which have often been utilized as high-performance ferroelectric or piezoelectric properties in ferroelectric oxides. These features suggest that there is a possibility of uniquely engineering the ferroelectric domains for organic electronic and optoelectric devices. The observation of both ferroelectricity and antiferroelectricity in the imidazole sequence can be related to the minute energy difference between their polar and antipolar arrangements. These findings recall the excellent electromechanical response in some piezoelectric lead zirconate titanate systems achieved by the electric-field-induced antiferroelectric to ferroelectric phase transition. It should also be noted that many imidazole-related compounds with various substituents are available from commercial sources and wellestablished chemical synthesis, and ferroelectric MBI is sold at low prices.

\section{Methods}

Preparation and calorimetry. All the purchased benzimidazoles were purified by recrystallization from alcohol (methanol, ethanol or 2-propanol) and/or temperature-gradient sublimation in vacuum until the coloured impurities were completely eliminated. Single crystals of MBI, DC-MBI and DFMBI were prepared by vacuum sublimation, whereas single crystals of TFMBI and TCMBI were grown by slow evaporation of the ethanol and 2-propanol solutions, respectively.

The thermal properties were examined using a differential scanning calorimeter (SII DSC6100) with the temperature calibration performed using the melting point of indium ( $429.8 \mathrm{~K})$. The sample was encapsulated in an aluminium pan and heated at a rate of $5 \mathrm{~K} \mathrm{~min}^{-1}$.

Electric measurements. All the electric measurements were taken from single crystals with gold-paste electrodes. The dielectric permittivity was obtained by measuring the capacitance with an LCR metre. The $P-E$ hysteresis curves were collected on a ferroelectrics evaluation system (Toyo Corporation, FCE-1) consisting of a current/charge-voltage converter (Toyo Corporation Model 6252), an arbitrary waveform generator (Biomation 2414B), an analogue-to-digital converter (WaveBook 516) and a voltage amplifier (NF Corporation, HVA4321). Both the dielectric hysteresis measurements and the modified double-wave method measurements were performed with a high-voltage triangular wave field and various alternating frequencies. The benzimidazole crystals were immersed in silicone oil to avoid electric discharge; the maximum electric field exceeded $30 \mathrm{kV} \mathrm{cm}^{-1}$. Hightemperature dielectric hysteresis measurements were conducted by heating the sample in the oil bath.

X-ray crystallographic analysis. The X-ray intensity data were collected on a Rigaku AFC7R four-circle diffractometer equipped with a Mercury CCD (chargecoupled device) area detector (graphite-monochromatized $\mathrm{MoK} \alpha$ radiation) for the DFMBI and DC-MBI crystals at room temperature. For the MBI, TCMBI and TFMBI crystals, the measurements at room temperatures were performed on a Rigaku DSC imaging plate system using Si-monochromatized synchrotron radiation $(\lambda=0.9995$ or $0.6878 \AA)$ at the BL- 8 A beamline of the Photon Factory, High Energy Accelerator Research Organization (KEK). All the calculations were performed using the CrystalClear, Rapid-AUTO and CrystalStructure crystallographic software packages of Molecular Structure Corporation and Rigaku Corporation. For the compounds investigated here, automatic symmetric analysis suggested a higher symmetry because symmetry lowering originates mainly from the locations of protons. The choice of a lowered symmetry gave chemically reasonable structures and was further justified by the present observations of ferroelectricity or antiferroelectricity. The final refinements were undertaken with anisotropic atomic displacement parameters for the non-hydrogen atoms and with isotropic parameters for the hydrogen atoms, except for those calculated on the aromatic moieties. CCDC-909439-909443 contains the supplementary crystallographic data for this paper. These data can be obtained free of charge from the Cambridge Crystallographic Data Centre via www.ccdc.cam.ac.uk/data_request/cif.

PFM measurements. The PFM measurements were carried out using a commercially available scanning probe microscope (Asylum MFP-3D) with 
a Ti/Ir-coated Si tip (Asylum Research). To achieve a good signal-to-noise ratio, we performed in-plane PFM measurements at $807 \mathrm{kHz}$, which is close to a torsional contact resonance frequency of the cantilever $(\sim 835 \mathrm{kHz})$. For imaging, an ac voltage of $1.3 \mathrm{~V}$ peak-to-peak was applied. To identify the direction of local polarization $\boldsymbol{P}_{\text {local }}$ in the MBI crystal, in-plane PFM images were collected under different cantilever geometries (Supplementary Fig. S6).

\section{References}

1. Lines, M. E. \& Glass, A. M. Principles and Applications of Ferroelectrics and Related Materials (Oxford University Press, New York, 1977).

2. Scott, J. F. Ferroelectric Memories (Springer-Verlag, Berlin, 2000).

3. Uchino, K. Piezoelectric Actuators and Ultrasonic Motors (Kluwer Academic, Dordrecht, 1997).

4. Shirane, G., Sawaguchi, E. \& Takagi, Y. Dielectric properties of lead zirconate. Phys. Rev. 84, 476-481 (1951)

5. Pan, W. Y., Zhang, Q. M., Bhalla, A. \& Cross, L. E. Field-forced antiferroelectric-to-ferroelectric switching in modified lead zirconate titanate stannate ceramics. J. Am. Ceram. Soc. 72, 571-578 (1989).

6. Horiuchi, S. \& Tokura, Y. Organic ferroelectrics. Nat. Mater. 7, 357-366 (2008).

7. Horiuchi, S. et al. Above-room-temperature ferroelectricity in a singlecomponent molecular crystal. Nature 463, 789-792 (2010).

8. Heredia, A. et al. Nanoscale ferroelectricity in crystalline $\gamma$-glycine. Adv. Funct. Mater. 22, 2996-3003 (2012).

9. Liu, Y., Zhang, Y., Chow, M.-J., Chen, Q. N. \& Li, J. Biological ferroelectricity uncovered in aortic walls by piezoresponse force microscopy. Phys. Rev. Lett. 108, 078103 (2012).

10. Horiuchi, S., Kumai, R. \& Tokura, Y. Hydrogen-bonding molecular chains for high-temperature ferroelectricity. Adv. Mater. 23, 2098-2103 (2011).

11. Brown, E. G. Ring Nitrogen and Key Biomolecules (Kluwer Academic Press, Dordrecht, 1998).

12. Grimmett, M. R. Imidazole and Benzimidazole Synthesis (Best Synthetic Methods) (Academic Press, London, 1997).

13. Barker, H. A. et al. Isolation and properties of crystalline cobamide coenzymes containing benzimidazole or 5,6-dimethylbenzimidazole. J. Biologic. Chem. 235, 480-488 (1960)

14. Freemantle, M. An Introduction to Ionic Liquids (RSC Publishing, Cambridge, 2009).

15. Walba, H. \& Isensee, R. W. Acidity constants of some arylimidazoles and their cations. J. Org. Chem. 26, 2789-2791 (1961).

16. Bureekaew, S. et al. One-dimensional imidazole aggregate in aluminium porous coordination polymers with high proton conductivity. Nat. Mater. 8, 831-836 (2009).

17. Katrusiak, A. Stereochemistry and transformations of $\mathrm{NH} \cdot \cdots \mathrm{N}$ hydrogen bonds Part I. Structural preferences for the hydrogen site. J. Mol. Struct. 474, 125-133 (1999)
18. Obodovskaya, A. E., Starikova, Z. A., Belous, S. N. \& Pokrovskaya, I. E. Crystal and molecular structure of 2-methylbenzimidazole. J. Struct. Chem. 32, 421-422 (1991).

19. Fukunaga, M. \& Noda, Y. New technique for measuring ferroelectric and antiferroelectric hysteresis loops. J. Phys. Soc. Jpn 77, 064706 (2008).

20. Balke, N. et al. Enhanced electric conductivity at ferroelectric vortex cores in BiFeO3. Nat. Phys. 8, 81-88 (2012).

21. Morimoto, M. \& Irie, M. Photochemical control of dielectric properties based on intermolecular proton transfer in a hydrogen-bonded diarylethene crystal. Chem. Commun. 47, 4186-4188 (2011).

22. Pająk, Z., Czarnecki, P., Szafrańska, B., Małuszyńska, H. \& Fojud, Z. Ferroelectric order in highly disordered molecular-ionic crystals. Phys. Rev. B 69, 132102 (2004).

23. Pajak, Z., Czarnecki, P., Szafrańska, B., Małuszyńska, H. \& Fojud, Z. Ferroelectric ordering in imidazolium perchlorate. J. Chem. Phys. 124, 144502 (2006).

24. Bordeaux, D. et al. New ferroelastic-ferroelectric compound: tanane. Phys. Rev Lett. 31, 314-317 (1973).

\section{Acknowledgements}

This work was partially supported by KAKENHI (no. 20110003) from MEXT and by the Japan Society for the Promotion of Science (JSPS) through the 'Funding Program for World-Leading Innovative R\&D on Science and Technology (FIRST Program) initiated by the Council for Science and Technology Policy (CSTP)'. The synchrotron X-ray study was performed with the approval of the Photon Factory Program Advisory Committee (nos 2009S2-003, 2011G643 and 2012G115).

\section{Author contributions}

S.H. prepared the samples and performed the dielectric measurements. F.K. and K.H. performed the PFM experiments. R.K., Y.M. and K.K. contributed to the diffraction studies, and S.H. and Y.T. conceived the study design and wrote the paper.

\section{Additional information}

Supplementary Information accompanies this paper on http://www.nature.com/ naturecommunications

Competing financial interests: The authors declare no competing financial interests

Reprints and permissions information is available at http://npg.nature.com/ reprintsandpermissions

How to cite this article: Horiuchi, S. et al. Above-room-temperature ferroelectricity and antiferroelectricity in benzimidazoles. Nat. Commun. 3:1308 doi: 10.1038/ncomms2322 (2012)

(c) (i) (2) This work is licensed under a Creative Commons Attributioncc. NonCommercial-ShareAlike 3.0 Unported License. To view a copy of this license, visit http://creativecommons.org/licenses/by-nc-sa/3.0/ 